Sports injuries

\section{Towards an evidence based prevention of sports injuries}

\section{R J Shephard}

\section{There are still obstacles to be overcome and challenges to be met}

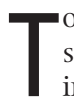
date, most initiatives to prevent sports injuries have been based on intuition rather than the solid experimental basis demanded by evidence based medicine. ${ }^{1}$ For example, since many eye injuries are sustained in activities such as ice hockey and squash, it is inferred that protection of the eyes and/or face should be required of all playing these sports. Likewise, because many serious head injuries are sustained by cyclists, it is argued that legislation should be enacted to enforce the use of protective helmets by cyclists.

Such initiatives are logical and appear to have substantial preventive value, but because of limitations in the design of existing experiments, the magnitude of any protection remains unclear. Clear proof of benefit requires a valid measure of exposure to risk, an appropriate assessment of the incidence and extent of injury, random assignment of subjects to a well controlled intervention, and a careful statistical assessment of any protection that is observed. For potential health benefits to be realized, there is also a need to communicate a synopsis of available information to the general public, and to convince them of the merits of any proposed preventive measures. ${ }^{2}$ These issues are here explored briefly, with particular reference to the use of helmets by cyclists.

\section{MEASURING EXPOSURE}

Most studies of sports injuries lack a clear measure of exposure to risk. The ideal statistic would be the average number of hours of involvement in a particular type and intensity of sport before an injury is incurred. However, injury rates vary widely between training and competition, and between recreation and high level competition. ${ }^{3}$ Thus, even if estimates of annual hours of sport participation are available, it is difficult to translate an injury rate per year into a meaningful rate of injury per hour of involvement in a given type and intensity of physical activity.

\section{INCIDENCE AND EXTENT OF INJURY}

Many studies simply report the number of injuries that are incurred by a group of athletes. However, lesions vary widely from minor trauma treated in an emergency room to major, life threatening events. Potential objective measures of the severity of injury range from the medical costs incurred and the days of work or schooling that are lost to decreases in quality adjusted life expectancy and death rates.

\section{RANDOMIZED TRIALS}

The most acceptable level of evidence in determining the efficacy of a proposed preventive measure is a randomized double blind controlled trial. ${ }^{1}$ It is almost impossible to realize this standard of proof in the context of sports injuries. For example, the wearing of any protective equipment such as a helmet is immediately obvious to both subject and observer. This is also true of most changes in the environment. Furthermore, it is almost impossible to recruit large numbers of volunteers for a non-blinded but randomized trial. At some future date, a quasiexperimental trial may be attempted through the distribution of protective equipment such as bicycle helmets to one group of schools, with a second well matched group of schools serving as controls. However, the findings from such a trial are likely to be compromised by a low rate of helmet use among the experimental group, and contamination leading to some helmet use by controls. relatively low, it will be necessary to recruit a very large number of subjects in order to demonstrate any significant effect from the preventive measures. benefits of cycle helmets seems limited to five case-control reports and several time series studies. ${ }^{4}$ In general, these analyses suggest the benefit of wearing a helmet when cycling. However, the nature of "cycling" is poorly defined. Some reports refer to competitive cycling, and these results are not necessarily applicable to recreational cycling using bicycles with upright handlebars. In children, many injuries also arise from trick behaviour rather than the proper and intended use of a bicycle. Further, because the rate of injury is

The currently available evidence on the

\section{COMMUNICATING BENEFIT}

Even after accumulating sound scientific evidence of benefit from a preventive tactic, it is not always easy to communicate this information to the general public. Sibert noted problems of communication in the context of cycle helmet use. ${ }^{2}$ Some individuals saw the wearing of a helmet as reducing the enjoyment of cycling and thus as discouraging a form of physical activity that was beneficial to health. ${ }^{5}$ One study based its calculations on the dubious assumption that all road incidents causing fatal head injuries would have been prevented if the individuals concerned had worn a helmet. Working from this premise, the authors concluded that more lives would have been saved if pedestrians and motorists had worn helmets than if such action had been taken by cyclists. ${ }^{6}$

At best, the wearing of a helmet is likely to reduce rather than abolish head injuries, and it will not make cycling entirely risk-free. Certainly, the use of helmets should not discourage the introduction of other preventive measures, such as the development of cycle paths and the introduction of "traffic calming" devices. Some authors have questioned the efficacy of cycle helmets (mainly on the basis of anecdotal evidence). ${ }^{7}$ It is clear that some helmets are much stronger, better designed and more effective than others, and unfortunately current data do not relate the extent of protection to the quality of the helmet that is worn. Case-control studies suggest that wearing a typical cycle helmet reduces the risk of a head injury to about one third of the control value, with a somewhat greater reduction in the incidence of more serious head injuries. ${ }^{78}$ Helmets are not cheap, and cost-benefit analyses are thus appropriate. In deprived areas, special measures may be needed to facilitate helmet use by poor children. ${ }^{9}$ In one study, the estimated cost per life saved was acceptable for primary schoolchildren (around NZ \$100 000), but it was very high for adults (around NZ \$1 000000$).^{7}$ Thus, the authors of this report concluded that cycle helmets should be recommended for children, but not for the average adult cyclist.

\section{CONCLUSIONS}

There are many obstacles to an evidence based programme of sports injury prevention. Nevertheless, modifications of questionnaires can enhance assessments of risk exposure, and define the nature and severity of injuries more clearly. In some situations, quasiexperimental assessments of protection are 
possible. But even when benefit has been categorically established, public acceptance and implementation of appropriate preventive measures will remain a major challenge.

Injury Prevention 2005;11:65-66.

doi: 10.1136/ip.2004.007963

Author's affiliations

R J Shephard, Faculty of Physical Education and Health and Department of Public Health Sciences, Faculty of Medicine, University of Toronto, Toronto, Ontario, Canada
Correspondence to: Professor Roy Shephard, PO Box 521, 41390 Dryden Road Brackendale, BC VON 1HO, Canada; royjshep@shaw.ca

\section{REFERENCES}

1 National Institutes of Health and National Heart, Lung and Blood Institute. Clinical guidelines on the identification, evaluation and treatment of overweight and obesity in adults: the evidence report. Obes Res 1998;6:51S-209S.

2 Sibert JR. Children and cycle helmets: the case for. Child Care Health Dev 1996;22:99-103.

3 Macintosh DI, Skrien TJ, Shephard RJ. Physical activity and injury. A study of sports injuries at the
University of Toronto, 1951-68. J Sports Med 1972; 12:224-37.

4 Thompson DC, Patterson MQ. Cycle helmets and the prevention of injuries. Recommendations for competitive sport. Sports Med 1998:25:213-19.

5 Carnall D. Cycle helmets should not be compulsory. BMJ 1999;318:1505.

6 Kennedy A. The pattern of injury in fatal pedal cycle accidents and the possible benefits of cycle helmets. Br J Sports Med 1996;30:130-3.

7 Hansen P, Scuffham PA. The cost-effectiveness of compulsory bicycle helmets in New Zealand. Aust J Public Health 1995; 19:450-4.

8 Lee AJ, Mann NP. Cycle helmets. Arch Dis Child 2003:88:465-6.

9 Kendrick D, Royal S. Lids for Kids project team. Cycle helmet ownership and use; a cluster rando mised controlled trial in primary school children in deprived areas. Arch Dis Child 2004;89:330-5.

\section{COCHRANE CORNER}

\section{Binge drinking}

T

he Cochrane Collaboration is an international network but the editorial base of the Cochrane Injuries Group (CIG) is in Britain and we are exposed to the British media's current "take" on injury prevention. Road traffic injuries may be the biggest killer butso we are told - the public worries more about violent crime.

One cause of violence that is currently under the spotlight is binge drinking. In recent years there has been a change in British drinking culture and some city centres-not to mention the centre of the small town where I live-can indeed resemble a battlefield on a Friday or Saturday night. However, the media revels in its exaggeration of the scale of the injury problem this causes, focusing inevitably on injuries from violent attacks rather than on unintentional injuries caused by drunks to themselves or others. Arguably, the long term effects on the health of binge drinkers, together with the damage to property and the waste of police and health service time, constitute a bigger problem than alcohol related injuries, whether intentional or unintentional.

Nevertheless, there is some cause for concern and the UK government's plans to further liberalise drinking legislation have added to public alarm, with encouragement from the media which warns that 24 hour drinking will result in unceasing mayhem on our streets.

Various answers to the problem of alcohol related violence have been proposed. These include health promotion campaigns targeted at drinkers and the prosecution of persistent offenders. There is also, however, a view that those who sell alcohol must bear some of the responsibility for any adverse consequences, particularly when the industry actively encourages the culture of excessive consumption at weekends and public holidays. What interventions to reduce alcohol related injury have been tried in the places where alcohol is sold, and have they been effective? My colleague Katharine Ker has recently embarked on a systematic review that addresses this issue. ${ }^{1}$ Although we must not let the media dictate our priorities, it is satisfying to be involved in research that addresses a hot topic!

Visitors to Britain often remark on the tendency of our media to condemn efforts by government to improve health and safety. The pejorative term "the nanny state" is currently much in vogue. A number of the interventions that have been assessed (or are being assessed) by CIG reviewers would be described, by some, as "nannying". Such interventions would include encouraging the wearing of cycle helmets, discouraging the use of babywalkers, home safety education, and community based approaches to injury prevention. We need, of course, to evaluate the evidence for "nannying" approaches coolly, indeed soberly, rather than dismiss them because they are deemed to be unfashionable by the media. Ultimately, we should aim to persuade those who write inflammatory articles in newspapers to base their pronouncements on evidence and not just on their own opinions.

P Chinnock

Managing Editor, Cochrane Injuries Group; paul.chinnock@lshtm.ac.uk

\section{REFERENCE}

1 Ker K. Interventions in the alcohol server setting for preventing injuries (protocol). Cochrane Database Syst Rev 2005 (in press). 